\title{
Femtosecond planar electron beam source for micron-scale dielectric wake field accelerator
}

\author{
T. C. Marshall \\ Department of Applied Physics, Columbia University, New York, New York 10027 \\ Changbiao Wang \\ Department of Physics, Yale University, New Haven, Connecticut 06520-8120 \\ J. L. Hirshfield \\ Department of Physics, Yale University, New Haven, Connecticut 06520-8120 \\ and Omega-P, Inc., Suite 100, 345 Whitney Avenue, New Haven, Connecticut 06511
}

(Received 2 October 2001; published 18 December 2001)

\begin{abstract}
A new accelerator, LACARA (laser-driven cyclotron autoresonance accelerator), under construction at the Accelerator Test Facility at Brookhaven National Laboratory, is to be powered by a $1 \mathrm{TW} \mathrm{CO}_{2}$ laser beam and a $50 \mathrm{MeV}$ injected electron pulse. LACARA will produce inside a $2 \mathrm{~m}, 6 \mathrm{~T}$ solenoid a $100 \mathrm{MeV}$ gyrating electron bunch, with $\sim 3 \%$ energy spread, approximately 1 psec in length with particles advancing in phase at the laser frequency, executing one cycle each $35 \mathrm{fsec}$. A beamstop with a small off axis channel will transmit a short beam pulse every optical cycle, thereby producing a train of about 30, 3.5 fsec long, 1-3 pC microbunches for each laser pulse. We describe here a novel accelerator, a micron-scale dielectric wake field accelerator driven by a $500 \mathrm{MeV}$ LACARA-type injector that takes the output train of microbunches and transforms them into a near-rectangular cross section having a narrow dimension of $\sim 10 \mu \mathrm{m}$ and height of $\sim 150 \mu \mathrm{m}$ using a magnetic quadrupole; these bunches may be injected into a planar dielectric-lined waveguide (slightly larger than the bunch) where cumulative buildup of wake fields can lead to an accelerating gradient $>1 \mathrm{GV} / \mathrm{m}$. This proposed vacuum-based wake field structure is physically rigid and capable of microfabrication accuracy, factors important in staging a large number of accelerator modules. Furthermore, the accelerating gradients it promises are comparable with those for plasma accelerators. A LACARA unit for preparing suitable bunches at $500 \mathrm{MeV}$ is described. Physics issues are discussed, including bunch spreading and transport, bunch shaping, coherent diffraction radiation from the aperture, dielectric breakdown, and bunch stability in the rectangular wake field structure.
\end{abstract}

DOI: $10.1103 /$ PhysRevSTAB.4.121301

PACS numbers: 41.75.Jv, 41.75.Lx, 41.75.Ht, 96.50.Pw

\section{INTRODUCTION}

New techniques for accelerating electrons or positrons using lasers promise very high accelerating gradients $(>1 \mathrm{GeV} / \mathrm{m})$. Plasma microstructures set up by intense laser pulses have offered the promise of large gradients [1], but are troubled by such issues as system efficiency and staging. Here we describe a system, a micron-scale dielectric wake field accelerator, which achieves similar gradients as in a plasma accelerator [2], while having better efficiency and possibly easier staging alignment. The energy source for the system is the microbunch current obtained from a $500 \mathrm{MeV}$ rf linac, a device capable of bunch energy recovery and high efficiency when operated with superconducting cavity structures. The system requires a TW-level $\mathrm{CO}_{2}$ laser, but uses it only for chopping the bunch train, not for acceleration. Finally, the system is made up of fixed dielectric structures of small dimension which surround the electron bunch in vacuum; these structures are designed to be excited by fields at optical/IR wavelengths. We outline a technique to prepare a train of bunches of electrons at high energy, each of which is approximately $1 \mu \mathrm{m}(3.5 \mathrm{fsec})$ in length and carries a few
$\mathrm{pC}$ of charge, and which can be used to excite wake fields in small dielectric structures to provide $\mathrm{GeV} / \mathrm{m}$ gradients for the acceleration of electron or positron bunches. Large wake fields may be built up in these structures, resulting in the storage of appreciable energy, by the superposition of wake fields from many phased drive bunches; these fields then may be used to accelerate a trailing test bunch to high energy.

Wake field accelerators [3-5] are generally attractive because no external source of energy (laser energy here) is used in the structure itself. No new lasers or rf sources are required for the proposed system. Although we study just one module here, staging is required to reach high energy. Because of the small dimensions, this structure must be fabricated and assembled precisely by microcircuittype methods. The small transverse dimension of the device permits a buildup of very high accelerating fields, $\sim \mathrm{GeV} / \mathrm{m}$; such large fields in a dielectric structure are possible because the tunneling-ionization breakdown field of the dielectric can become large when exposed to femtosecond-duration pulses of high field [6]. Furthermore, a rectangular dielectric slab structure has the advantages that it can pass more beam charge, store more 
energy, and has better stability to transverse beam deflection than a cylindrical structure of comparable transverse dimension [7].

Section II of this paper presents a method of preparing femtosecond duration, planar $500 \mathrm{MeV}$ bunches, and Sec. III describes some of the physics issues of this novel wake field accelerator concept.

\section{PREPARATION OF FEMTOSECOND DURATION HIGH ENERGY BUNCHES}

Haimson and Mecklenburg [8] describe a beam chopper, where a microwave cavity operating in the $\mathrm{TM}_{110}$ mode is used to deflect an electron beam up and down across a collimator so as to transmit bunches. The bunches are spaced by a half-period of the microwave and have widths about one-tenth of that, so this type of chopper is not suitable for production of femtosecond bunches. Techniques have been developed recently to prepare short electron bunches by dynamic bunching, using an inverse free electron laser accelerator (IFEL). This device, which is powered by a $\mathrm{CO}_{2}$ laser, can bunch electrons on a scale that is a fraction of the laser wavelength. Such bunches are already in use in a staged $\mathrm{CO}_{2}$ laser IFEL project at the Accelerator Test Facility (ATF) at Brookhaven National Laboratory [9]. However, another $\mathrm{CO}_{2}$ laser accelerator is under construction by Omega-P at ATF, the LACARA (laser-driven cyclotron autoresonance accelerator) [10], which will become operational in 2002. Previous work showed that the electron bunch formed by LACARA can be chopped so as to prepare a train of $\sim \mathrm{pC}$ bunches $\sim 1 \mu \mathrm{m}$ in length and spaced by the laser wavelength [10]. LACARA can prepare this bunch train more accurately and reproducibly than with the IFEL, because the chopping depends only upon the geometry of a fixed chopper target in the beam line, rather than on the less precise longitudinal dynamical bunching process induced by the laser in the IFEL. In addition to using the upgraded TW $\mathrm{CO}_{2}$ laser facility at ATF, LACARA also utilizes a $6 \mathrm{~T}$ superconducting solenoid which permits a near-resonant interaction that can accelerate the entire kA electron bunch $(\sim 1 \mathrm{nC}$ in $\sim 1 \mathrm{psec})$. The injection energy is about $50 \mathrm{MeV}$, and the output energy of this version of a LACARA accelerator can be as high as $100 \mathrm{MeV}$ with an energy spread of $\sim 3 \%$, when using a $1 \mathrm{TW} \mathrm{CO}_{2}$ laser. In the prior analysis [10], the electron beam was assumed to be launched within an axial resonant magnetic field, and the beam was significantly accelerated. But in a working device, the electron beam must traverse a transition region first, where the guide field increases from zero to the required level. Simulations show that the transition region may increase the beam's rms emittance considerably, and thereby affect the quality of the accelerated/chopped bunch output. In this section, realistic injection of the bunch into the magnetic field is assumed. We then show the feasibility of the LACARA chopper for preparation of $500 \mathrm{MeV}$ femtosecond duration bunches, where the laser power is used only for chopping the bunch train, not for acceleration.

An attractive feature of a wake field structure excited by a train of bunches is illustrated in Fig. 1, a result of analysis for a microwave-scale planar dielectric wake field accelerator [4]. Here, ten drive sheet bunches spaced by $10.5 \mathrm{~cm}$ pass along the axis of two planar dielectric slabs (alumina) having outer conducting surfaces. The structure is designed such that many TM-like guided modes are excited by the short bunches, and such that the fundamental period of the modes equals the period of the bunch train. The combination of short bunch length and high dielectric constant material favors the creation of sharply localized pulses of longitudinal electric field. Wake fields from one bunch in a train can be superimposed upon the wake fields from prior bunches: this cumulative effect is shown in Fig. 1. Placement of a test bunch one-half period behind the last drive bunch will allow much higher acceleration gradients than with only one bunch. The gradient produced may not exceed what can be achieved with a single bunch having a total charge equal to the sum of the charges of all the drive bunches; however, it may well be easier to create a train of bunches of moderate charge and high quality than to do so with a single bunch of large charge. A comprehensive theory of wake fields in cylindrical dielectric structures [3] and analysis of stability for this geometry [11] have both been recently published. It turns out that the use of the planar geometry (in 3D geometry rather than the 2D example of Fig. 1), while not practical for microwave accelerator structures, is attractive when one considers the use of micron-long, tall rectangular cross section sheetlike bunches which could excite a 3D rectangular dielectric wake field structure having micronscale dimensions. In Fig. 2 is shown a schematic of a short rectangular electron bunch traveling between two dielectric layers: the width $2 a$ of the channel is $\sim 10 \mu \mathrm{m}$, the thickness $b-a$ of each dielectric layer is $\sim 10 \mu \mathrm{m}$, the

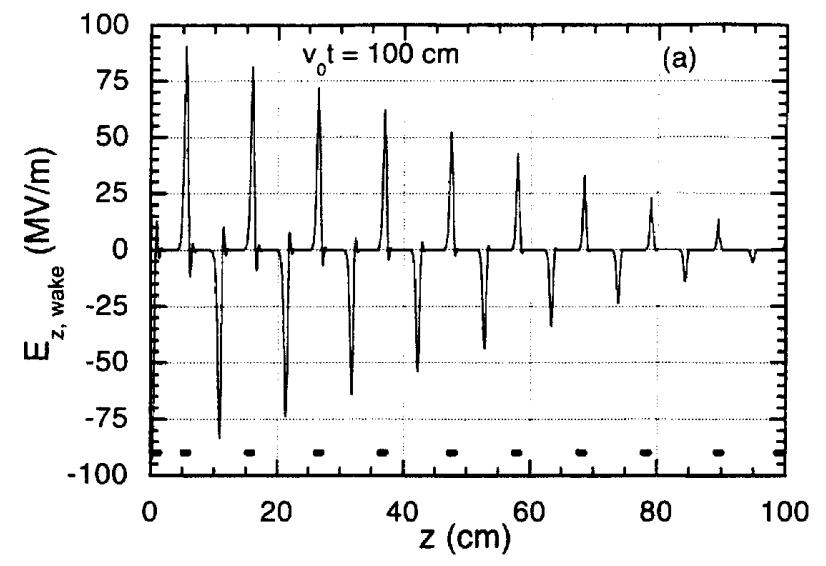

FIG. 1. Cumulative wake field set up by ten identical successive sheet bunches in a tuned microwave-scale dielectric slab structure, at the time the first bunch has moved $100 \mathrm{~cm}$ along the structure. Bunch locations are shown by black dots at the bottom of the figure. 


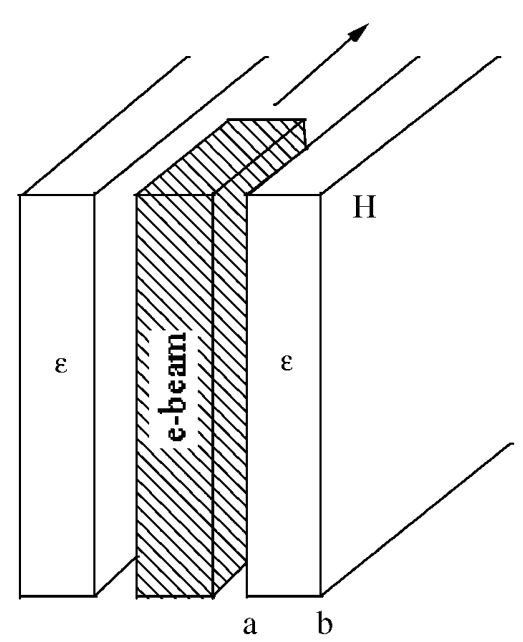

FIG. 2. Schematic of slab bunch within a planar optical wake field structure.

length is several $\mathrm{cm}$, and the height $H$ is at least 10 times the width. When a tall "sheet" bunch is injected into such a structure, LSE (longitudinal-section electric) and LSM (longitudinal-section magnetic) modes are excited [12]; but for a sufficiently tall structure, it is expected that the results will approach the performance of the $2 \mathrm{D}$ planar device. This occurs because the LSE modes become weak as the 3D structure height is increased, and the LSM modes will approximate the TM modes of the planar device.

A conceptual schematic of a LACARA device that will form the short bunches is shown in Fig. 3. Noteworthy are the beamstop, the beam focus/defocus quadrupole, and the dielectric wake field structure described above. For a brief summary of the physics involved in LACARA, we mention that a resonance condition exists which connects the laser frequency $\omega_{L}$ to the value of solenoidal magnetic field (expressed in terms of the rest electron gyrofrequency $\Omega_{0}$ ) and the particle energy,

$$
\Omega_{0}=\gamma \omega_{L}\left(1-n \beta_{z}\right),
$$

where the index of refraction $n(r=0, z)=\left(c k_{z} /\right.$ $\left.\omega_{L}\right)_{r=0}=\nu_{\mathrm{gr}} / c$, where $\nu_{\mathrm{gr}}$ is the axial group velocity, and the phase velocity is $\omega_{L} / k_{z}$. The index $n(r, z)$ is written in terms of an effective wave number that depends on an axial $k_{z}$, a radial $k_{r}$, and (for synchronism) the particle velocity ratio $\nu_{r} / \nu_{z}$. The laser fields are obtained for a Gaussian mode, and all components of $E_{L}(r, \theta, z)$, $B_{L}(r, \theta, z)$, and the components of $k$ are related by the constant phase condition of the wave $d \psi / d t=0$. The electrons are injected into the interaction region with random phases, but they are rapidly phase trapped and then accelerated. The electrons receive transverse momentum from the laser field, and the energy exchange is via $v_{\perp} E_{L}$. Relativistic equations of motion of particles in the fields allow solutions for the orbits; at each stage of the calculation, the solenoidal field is set to be in resonance to maximize acceleration.

At ATF, the first LACARA is to be operated as an accelerator as described above. However, for the application of making short, rectangular-profile bunches of micron dimensions suitable for wake field acceleration in an "optical" structure, it is necessary to achieve an input bunch to LACARA that has very small emittance. In the following example, this was taken to have a normalized emittance value of $1 \mathrm{~mm}$ mrad at an energy of $500 \mathrm{MeV}$. This emittance is believed to be within the capability of state-ofthe-art rf linacs [9], and the higher energy is necessary when staging a sequence of wake field structures for accelerating a collinearly moving test bunch to very high energy. In this application, the LACARA is not used so much as an accelerator, but rather as a phase buncher. In the example given, the solenoidal field is $1.76 \mathrm{~T}$ and is $5 \mathrm{~m}$ in length, and a $5 \mathrm{TW} \mathrm{CO}_{2}$ Gaussian profile laser beam is introduced along the axis of the magnetic field. The bunch charge is taken to be $1 \mathrm{nC}$ in $1 \mathrm{psec}$, and the input bunch radius is taken to be $30 \mu \mathrm{m}$.

In Fig. 4 is shown the acceleration of this bunch of electrons, seen to be quite small. (In fact, only $1 \mathrm{~mJ}$ of energy has been extracted from the $5 \mathrm{~J}$ laser pulse in the example discussed; this permits the laser to be periodically refocused to excite many modules in a staged system.) At the end of the interaction region where the axial magnetic field is very weak, the electrons are distributed about an annular ring having radius and thickness depending on the emittance and beam spot size at the input. Figure 5

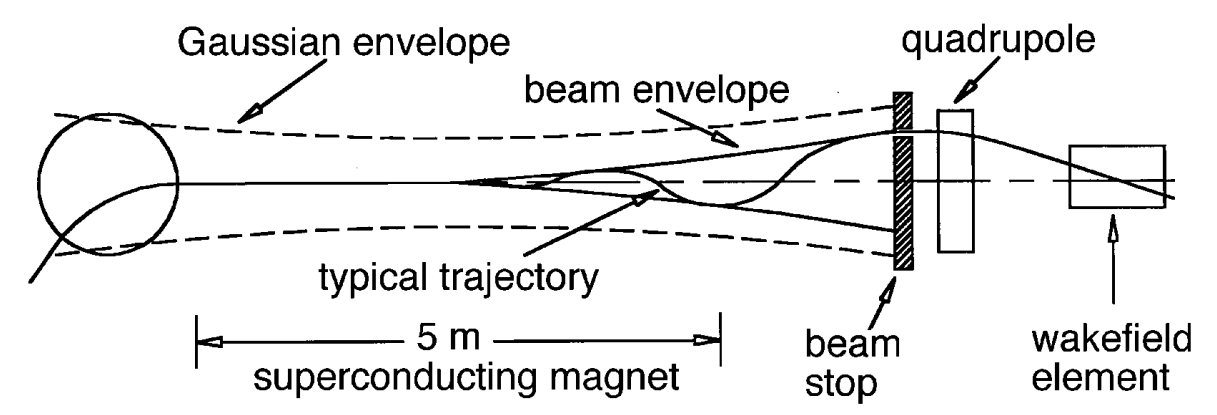

FIG. 3. Schematic of LACARA with beamstop inserted to produce a chopped beam that is formed into a sheetlike transverse profile using a quadrupole, for generation of intense wake fields in a planar dielectric accelerating structure. Radial scale is expanded for clarity. 


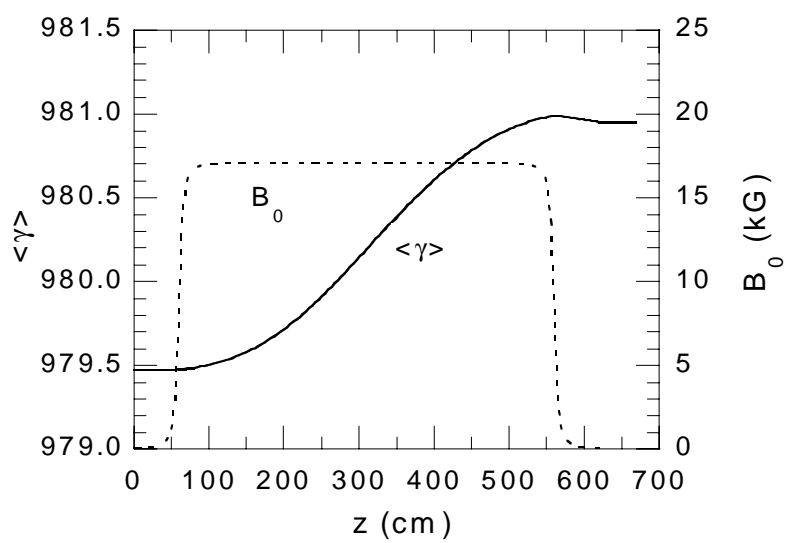

FIG. 4. Magnetic field profile and gain of energy of an electron bunch in LACARA, using a 5 TW circularly polarized $\mathrm{CO}_{2}$ laser in a Gaussian beam with $266.7 \mathrm{~cm}$ Rayleigh range. The superconducting magnet lies between $z=60$ and $560 \mathrm{~cm}$; the beamstop extends from $z=620$ to $625 \mathrm{~cm}$; the quadrupole is located between $z=630$ and $635 \mathrm{~cm}$.

shows the particle distribution in the $(x-y)$ plane at the end of the device; the motion of the electrons is such that arriving particles have successive loci that circulate around the $(x-y)$ plane at an angular frequency that corresponds to the 35.3 fsec period of the $10.6 \mu \mathrm{m}$ wavelength of the laser that drives the LACARA. Figure 6 shows how the particle distribution varies periodically in $x$ (with the laser period) at the plane of the beamstop. This plane, located at $z=620 \mathrm{~cm}$, is also the front face of a $5 \mathrm{~cm}$ thick target beamstop, which contains a small hole for transmitting a fraction of the electrons, shown at the output end of the beamstop at $z=625 \mathrm{~cm}$ (Fig. 7). The hole limits transmission of an angular portion of the beam profile at the

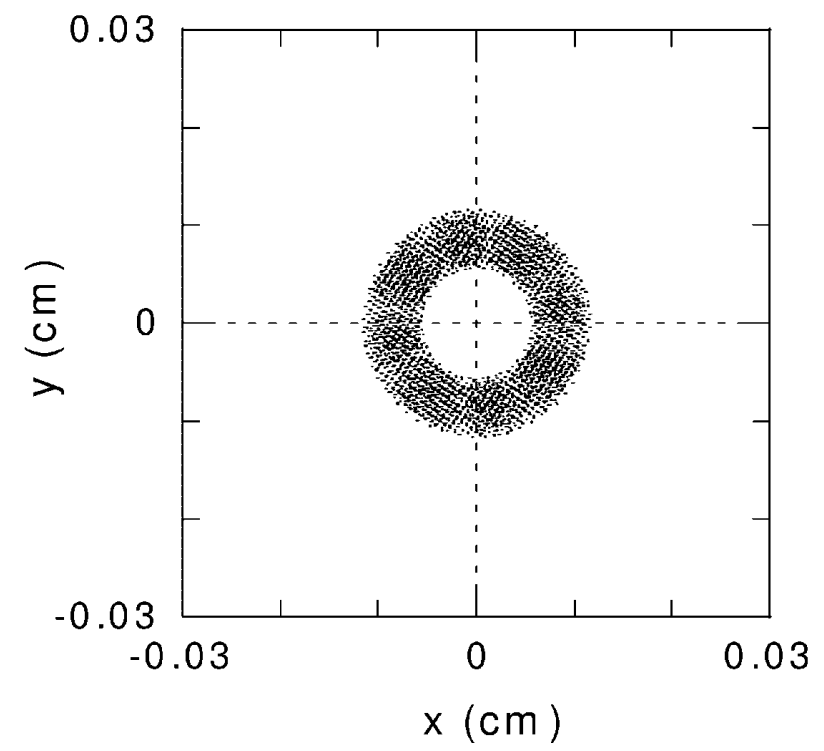

FIG. 5. Particle distribution in the $x-y$ plane at $z=620 \mathrm{~cm}$ at the end of LACARA. The input bunch radius is $30 \mu \mathrm{m}$, and the input beam normalized emittance is $1 \mathrm{~mm} \mathrm{mrad}$.

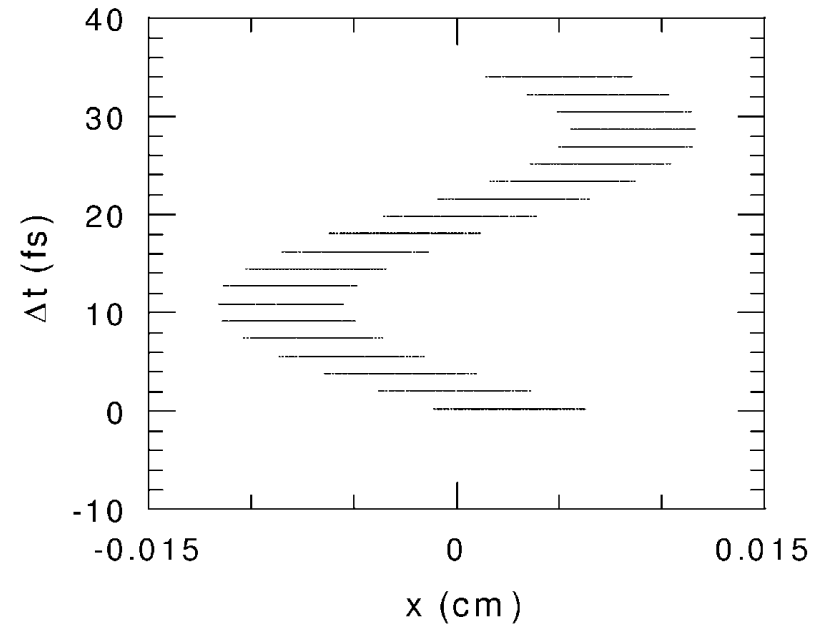

FIG. 6. Particle distribution $\Delta t$ vs $x$ at $z=620 \mathrm{~cm}$.

beamstop, and is dimensioned so that the electrons transmitted through it form a pulse of about $3.5 \mathrm{fsec}$ in duration (10\% of a cycle). The hole in the beamstop target also functions as an emittance "filter." The transmitted microbunch charge, for an average current of $1000 \mathrm{~A}$ in the full bunch, is $3.5 \mathrm{pC}$.

The microbunches transmitted by the hole in the beamstop (containing $\sim 2 \times 10^{7}$ electrons; see Fig. 8 ) could be used for applications such as creation of very short pulses of $x$ rays using Compton backscattering off a laser pulse, or injection into the wavelength-scale plasma wake fields set up by the same laser that powers LACARA (thereby maintaining a fixed phase reference for accelerating the injected electrons). However, in what follows, we describe an application in which the emitted chopped bunches are used to set up wake fields in a tall planar dielectric structure,

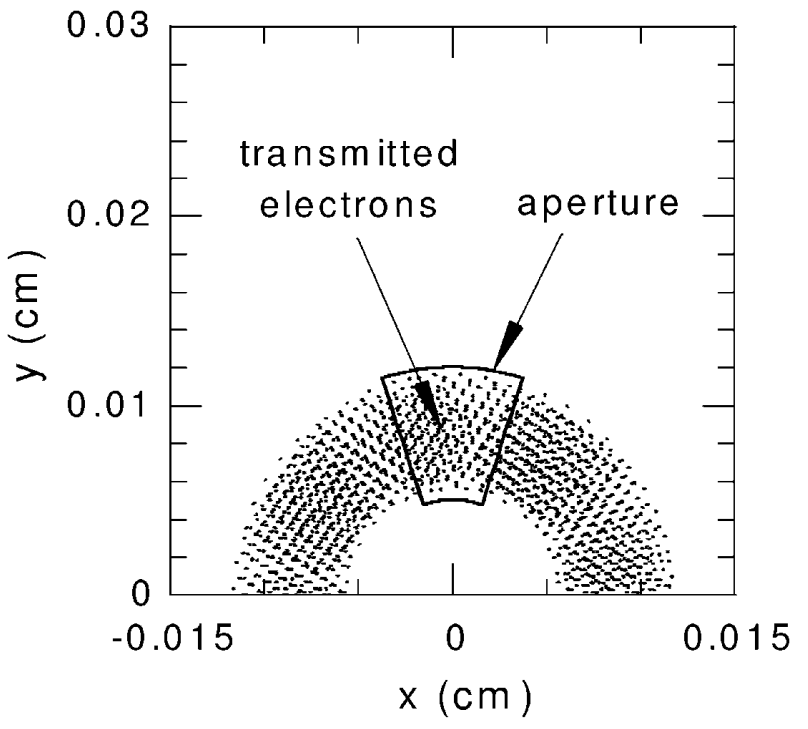

FIG. 7. Particle distribution in the $x-y$ plane at $z=625 \mathrm{~cm}$ (back of beamstop). The aperture in the beamstop also functions as an emittance filter. 


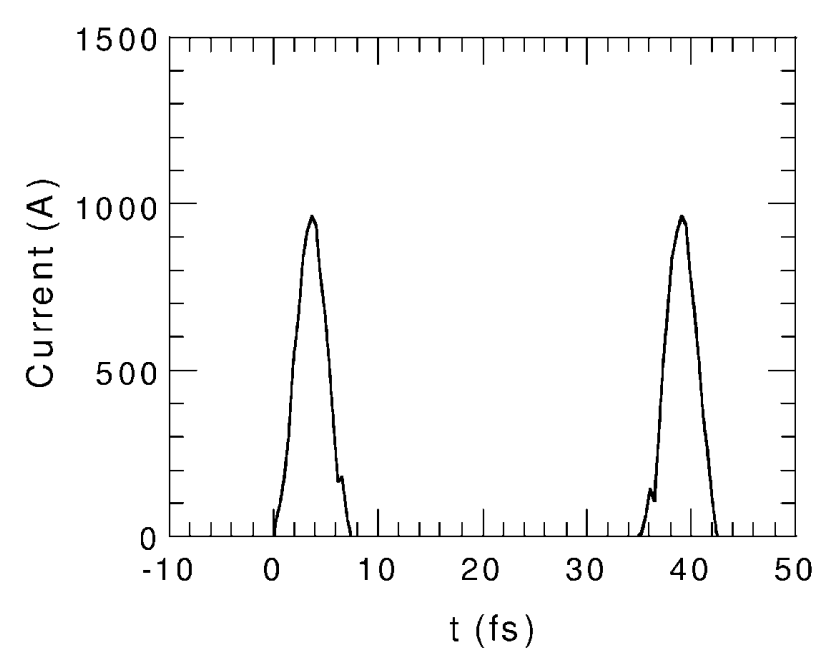

FIG. 8. Current transmitted by the beamstop aperture versus time at $z=625 \mathrm{~cm}$.
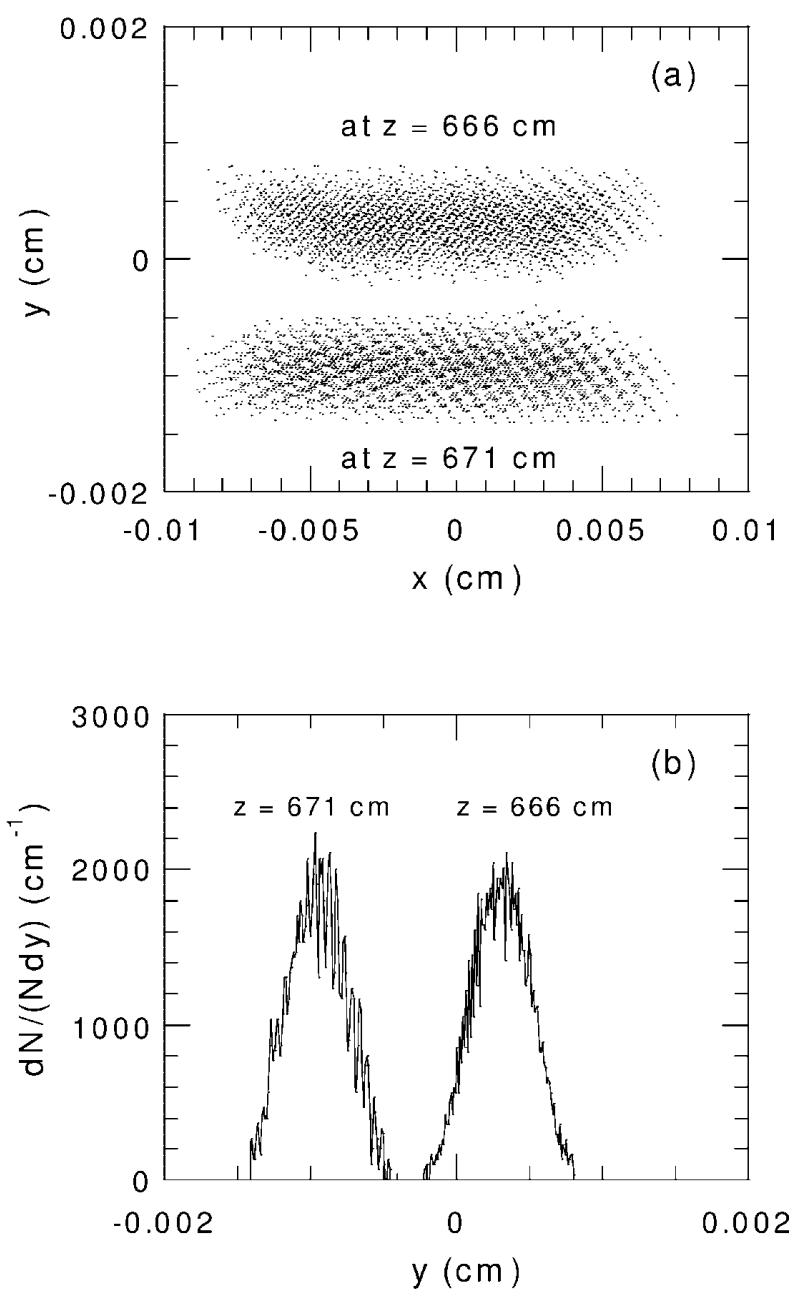

FIG. 9. Sheetlike beam profiles (a), and narrow-dimension distribution functions (b), following the quadrupole, at $z=666$ and $671 \mathrm{~cm}$. Note that the $x$ and $y$ scales are different in (a). which could be regarded as one modular component out of many for an advanced concept linear accelerator with acceleration gradient $\sim \mathrm{GeV} / \mathrm{m}$, that could be used to produce very high energy bunches. (The number of modules would be of the order of 1000 for a TeV accelerator using $500 \mathrm{MeV}$ drive bunches.)

In Fig. 3, a quadrupole focus/defocus element is shown beyond the beamstop that is to prepare a sheetlike beam profile. The quadrupole is located between $z=630$ and $635 \mathrm{~cm}$, and distorts the bunch into a sheetlike profile at $z \sim 666 \mathrm{~cm}$; the distribution and profile of the electron bunch are shown in cross section in Fig. 9. The quadrupole magnet produces a pole-face field of $1.05 \mathrm{~T}$ with pole radius of $1 \mathrm{~cm}$, and has its axis located at $x=y=0$. The narrow transverse dimension of the bunch is seen to be $10 \mu \mathrm{m}$, and the wide one is about $160 \mu \mathrm{m}$ (the axial length of the bunch is $1 \mu \mathrm{m}$ ). These bunch dimensions persist for a distance $>10 \mathrm{~cm}$ along the axis, which defines the maximum length of the wake field structure.

\section{PHYSICS ISSUES OF THE WAKE FIELD DEVICE}

The choice of a slab beam is motivated by two considerations. First, a slab-shaped electron bunch inside a rectangular dielectric-slab structure has the useful property that an infinitely tall bunch is entirely stable against breakup by transverse wake fields [7]; further, if the bunch has finite height, stability is much improved compared with that of a cylindrical channel passing comparable bunch charge. To say this somewhat differently, the improvement in stability occurs because the slab channel is passing lower bunch charge per unit height than the comparable cylindrical channel. The transverse wake field coupling drops from $\sim\left(\omega_{L} / c\right)^{3}$ in the cylindrical case to $\sim\left(\sigma_{x}\right)^{-3}$ in the rectangular geometry [7]. Thus, transmission through small structures can be assured over distances approximately several $\mathrm{cm}$ so that correctly phased bunches will pick up or deposit appreciable energy in one stage. Stability of bunch motion in accelerators is always a fundamental issue, and by choosing a tall rectangular cross section one can improve stability while achieving high gradient in the small structure. Of course, stability of an extended system of staged modules could require some external focusing, but this issue is beyond the scope of the present work.

Second, our prior work with dielectric wake field accelerator structures [4] has shown that a short charge bunch can excite a high-amplitude well-defined periodic train of axial electric field pulses that trail the bunch on axis. An example for a $2 \mathrm{D}$ rectangular microwave structure using alumina as a dielectric is shown in Fig. 1; it is essential that the dielectric be free of dispersion in order that the fields from the many TM modes excited by the bunch may be exactly superimposed. In the case of the infinite (2D) dielectric slab structure (Fig. 1), the structure itself is nearly free of dispersion (unlike the example of cylindrical cross 
section). To what extent a 3D rectangular structure of finite height - albeit with aspect ratio $\sim 20$ - approaches the desirable dispersion-free quality of the infinitely tall structure remains an objective of further study. However, this much we can say: in the 3D structure are set up LSE and LSM modes. As the structure becomes very tall and narrow, the LSM dominate, and their dispersion relation becomes the same as the 2D slab in the limit of infinite height. Also, the frequency spacing of the LSM modes becomes nearly constant (and thus the modes are evenly spaced) for height/width ratio $>20$. Thus we expect that the wake fields set up by a bunch in this type of tall rectangular structure will also show the rather clean structure obtained in Ref. [4]. The dispersion relation for the modes in the 2D limit yields an expression for mode frequencies $\omega_{m}=(m+1 / 2) \Delta \omega$ separated by a constant frequency $\Delta \omega$, where $m$ (an integer) is the mode index, and where

$$
\Delta \omega=(\pi \beta c)(b-a)^{-1}\left(\kappa \beta^{2}-1\right)^{-1 / 2} .
$$

For a train of bunches separated by $10.6 \mu \mathrm{m}$, an appropriate choice of dimensions is $2 a \sim 10 \mu \mathrm{m}$ and $b-a \sim$ $10 \mu \mathrm{m}$ for dielectric constant $\kappa \sim 3.5$. The Coulomb field of the charge, which is of the same order as the peak wake field $E_{z}$ following a $3.5 \mathrm{pC}$ bunch that is $160 \mu \mathrm{m}$ tall, is then $\sim Q_{x} / 2 a \varepsilon_{0}$ where $Q_{x}$ is the bunch charge per unit height $(\mathrm{C} / \mathrm{m})$ of the slab bunch; this is $\sim 0.2 \mathrm{GV} / \mathrm{m}$ for just one bunch. From this example, one can appreciate that it is the small size of the structure, intrinsic to the use of laser wavelengths, that favors high accelerating gradients. However, the axial electric field may be increased further by the superposition of several following bunches, each spaced by $10.6 \mu \mathrm{m}$, obtained from the original $1 \mathrm{psec}$ (300 $\mu \mathrm{m}$ long) bunch that was prepared by the LACARA. The maximum field strength that can be developed by wake field superposition is limited by the breakdown field in the dielectric. But we point out that in the wake field device this is determined by the $\sim 5$ fsec time scale from the passage of a microbunch near an adjacent region of dielectric, and therefore the breakdown field could exceed $3 \mathrm{GV} / \mathrm{m}$ [6]. (It is instructive to note that the rectangular structure will pass more bunch charge with lower field strength at the dielectric than will the cylindrical structure.) The length of the wake field structure that would contain several "drive" bunches would be only $1 \mathrm{psec}$, or $0.3 \mathrm{~mm}$; however, the actual length would be set by energy loss rate of the last bunch, $\sim 3 \mathrm{GV} / \mathrm{m}$ in the example, and therefore a structure $\sim 10-20 \mathrm{~cm}$ in length would be appropriate.

In order to study such accelerating structures, we must be able to transport the chopped electron bunch through the beam shaping apparatus to a location where the beam can be analyzed (e.g., by transition radiation) or where it is accelerated in a wake field structure. The very short dimensions of the bunch suggest that spreading along the direction of motion from the space charge field might be significant. The electric field at the forward or trailing edges of the bunch can be estimated from Gauss's law as
$Q / 2 A \varepsilon_{0}$, where $A$ is the cross-sectional area of the bunch and $Q$ is the total bunch charge. One may then consider the motion of an electron at the front and at the rear end of the bunch, which move away from the center by Coulomb repulsion. The pulse length increment $\Delta z=z_{\text {front }}-$ $z_{\text {back }}-2 L$, where $2 L$ is the initial bunch length, grows in time $t$ on the $z$ axis of symmetry approximately as

$$
\Delta z \approx e Q t^{2} / 2 \varepsilon_{o} A m_{0} \gamma^{3},
$$

where $e$ and $m_{0}$ are the electron charge and the rest mass, respectively, and $\gamma$ is the relativistic energy factor. The bunch lengthening effect is quite small on the scale of a single module, and a distance $\sim 20 \mathrm{~m}$ is seen to be required to double the bunch length. For comparison, if the $500 \mathrm{MeV}$ beam energy spread was $(\delta \gamma / \gamma)_{z}=0.1 \%$, the resulting spread of longitudinal velocities $\delta \nu_{z}=$ $\left(c / \gamma^{2}\right)(\delta \gamma / \gamma)_{z}$ would double the bunch length in $\sim 1 \mathrm{~km}$. However, the space charge calculation is admittedly crude since the actual spreading depends on the geometry of the cross section and the actual charge distribution. For this, a study of pulse spreading using a particle-in-cell code is necessary; however, our estimate suggests that the chopped pulse can be processed and transported to a remote location without the use of plasma for neutralization.

A problem that affects all device structures in which a highly relativistic electron bunch must pass through an aperture is "coherent diffraction radiation" [13], a form of transition radiation in which some of the Poynting flux associated with the moving bunch is intercepted by the dielectric or metal structure and thereby lost from the bunch. In Gaussian cgs units, the ratio of the energy lost by the bunch to its incident bunch energy $\sim \gamma N m_{0} c^{2}$ for the rectangular slab device is

$$
\frac{1}{16 \pi^{2} m_{0} c^{2}} \frac{N e^{2}}{H} \ln \left(\frac{H}{a}\right) \sim 10^{-5},
$$

where $a$ and $H$ are, respectively, the half-width and the height of the aperture. This estimate shows that coherent diffraction radiation presents no serious obstacle for an accelerator up to TeV energy, since only about $10 \mathrm{MeV}$ would be lost to diffraction radiation per stage. However, if a single bunch were to be used having charge equal to that of the full train of 30 microbunches, then its diffraction radiation might amount to $300 \mathrm{MeV}$ per stage, clearly an intolerable magnitude.

Wake field structures are attractive because no external source of energy is used to excite the structure itself. Thus no new source of energy is needed for the optical wake field accelerator structure, other than the $\mathrm{CO}_{2}$ laser source that generates the microbunches in LACARA. The beam could be refocused into sheet configuration down stream periodically by quadrupole magnets located between the staged wake field accelerator units. The breakdown limit of the wake field structure is not determined by the slow filling time of the structure by electromagnetic 
energy, but rather by the much shorter time of the passing field pulses set up by the short (micro)bunches. The breakdown problem in connection with rf energy slowly filling the cavities of a conventional linac also applies to optical energy slowly filling an optical Fabry-Pérot resonator [14]. The use of a train of femtosecond bunches to build up the accelerating field in an "optical" wake field structure should have superior breakdown field properties to the optical resonator, and also should be competitive with the laser-wake-field plasma accelerator which is under intensive investigation. The proposed vacuum-based wake field structure can be mechanically rigid and therefore should be a more reproducible and controllable element in a staged system than would be an array of pulsed plasma elements. Further work must be done to identify and develop broadband transparent, high dielectric constant materials suitable for these optical-scale wake field structures, as well as to develop a transport lattice for multistage operation. The structure is capable of microfabrication accuracy, an important consideration when staging a large number of modules. Finally, the charge of a sequence of drive bunches can be programmed [15] so as to increase progressively according to a certain relationship [16], permitting a considerable increase in the transformer ratio - the ratio of the maximum energy gain behind the drive bunch to the maximum energy loss inside the drive bunch [15].

\section{ACKNOWLEDGMENTS}

The authors would like to thank Dr. M. A. LaPointe for his help in the preparation of the manuscript. This work was supported by the Department of Energy.
[1] E. Esarey, P. Sprangle, J. Krall, and A. Ting, IEEE Trans. Plasma Sci. 24, 252 (1996).

[2] P. Sprangle, B. Hafizi, J. R. Penano, R. F. Hubbard, A. Ting, C. I. Moore, D. F. Gordon, A. Zigler, D. Kaganovich, and T. M. Antonsen, Jr., Phys. Rev. E 63, 056405 (2001).

[3] S. Y. Park and J.L. Hirshfield, Phys. Rev. E 62, 1266 (2000).

[4] T-B. Zhang, J. L. Hirshfield, T. C. Marshall, and B. Hafizi, Phys. Rev. E 56, 4647 (1997).

[5] T. C. Marshall, J-M. Fang, J. L. Hirshfield, and S. Y. Park, in Advanced Accelerator Concepts: Ninth Workshop, edited by P. Colestock and S. Kelley, AIP Conf. Proc. No. 569 (AIP, New York, 2001), p. 316.

[6] P. Sprangle, B. Hafizi, and R. F. Hubbard, Phys. Rev. E 55, 5964 (1997).

[7] A. Tremaine, J. Rosenzweig, and P. Schoessow, Phys. Rev. E 56, 7204 (1997).

[8] J. Haimson and B. Mecklenburg, in Proceedings of the Particle Accelerator Conference, Chicago, 1989 (IEEE, New York, 1989), p. 243.

[9] W. D. Kimura et al., Phys. Rev. Lett. 86, 4041 (2001).

[10] J. L. Hirshfield and C. Wang, Phys. Rev. E 61, 7252 (2000).

[11] S. Y. Park and J.L. Hirshfield, Phys. Plasmas 8, 2461 (2001).

[12] R. E. Collin, in Field Theory of Guided Waves (IEEE, New York, 1991).

[13] M. Castellano et al., Phys. Rev. E 63, 056501 (2001).

[14] J. Rosenzweig and P. Schoessow, in Advanced Accelerator Concepts: Eighth Workshop, edited by W. Lawson, C. Bellamy, and D. Brosius, AIP Conf. Proc. No. 472 (AIP, New York, 1999), p. 693; see also J. Rosenzweig, A. Murokh, and C. Pellegrini, Phys. Rev. Lett. 74, 2467 (1995).

[15] K. L. Bane, P. Chen, and P. B. Wilson, IEEE Trans. Nucl. Sci. 32, 3524 (1985).

[16] J. G. Power, W. Gai, and A. Kanareykin, in Advanced Accelerator Concepts: Ninth Workshop (Ref. [5]), p. 605. 